\title{
VARIABILITY IN THE MILLIMETER AND RADIO DOMAINS
}

\author{
ESKO VALTAOJA \\ Metsähovi Radio Research Station, Helsinki University of Technology, SF-02540 Kylmälä, \\ Finland
}

\section{Introduction}

The radio domain spans only a small fraction of the total electromagnetic spectrum, and is for most active galaxies energetically insignificant in comparison with higher frequencies.However, only in the radio regime can we obtain continuous fluxcurves and be certain that we are not missing anything. We also believe we know where the radiation comes from, and by which mechanisms it is produced. Furthermore, since VLBI generally cannot resolve the smallest core components, multifrequency continuum monitoring remains our only way to study the innermost radio cores of AGN. Finally, continuum monitoring is cheap (although timeconsuming) in comparison with most other methods, and therefore we can observe many more sources and with far better time coverage.

The bulk of high radio frequency data comes from two dedicated monitoring programs. At the University of Michigan, 60-200 sources have been observed regulary at 4.8, 8 and $14.5 \mathrm{GHz}$ since 1965 (Aller et al 1985). At the Metsähovi Radio Research Station, some 50-100 sources are being monitored mainly at 22 and 37 $\mathrm{GHz}$, with over 20000 flux measurements obtained since 1980 (Teräsranta et al. 1992). Both programs are now providing well sampled fluxcurves of the brightest compact northern and equitorial radio sources.

At still higher frequencies observations become increasingly difficult, and there are no similarly dedicated telescopes. We have used the SEST telescope in Chile since 1987 for persistent monitoring of 20-50 mainly equatorial sources at 90 and $230 \mathrm{GHz}$ (Tornikoski et al. 1993). At IRAM, a number of bright quasars are used for pointing checks (Steppe et al. 1992) at 90, 150 and $230 \mathrm{GHz}$. Finally, the JCMT at Hawaii has been used for $\mathrm{mm} / \mathrm{sub}-\mathrm{mm}$ monitoring of about $20 \mathrm{AGN}$ (Gear et al. 1993). There are also occasional observations with other millimeter telescopes. However, even with combined data from all these the variations at $90 \mathrm{GHz}$ or higher are woefully undersampled.

\section{Questions for the radio domain}

Radio observations are an essential part of multifrequency studies. But what questions can we attempt to solve using only radio data? We want to know where the radiation comes from. We want to know what mechanisms are producing it, and whether they are similar in all AGN. For the mechanisms, the paradigm to be

T. J.-L. Courvoisier and A. Blecha: Multi-Wavelength Continuum Emission of AGN, 145-154.

(C) 1994 IAU. Printed in the Netherlands. 
tested is the shocked relativistic jet scenario. For the differences and similarities we are starting to look increasingly hopefully at unified models of AGN.

\subsection{Origin OF The RADIO FLUX}

Essentially all radio flux and variations seem to originate from just three different synchrotron components. Below $10 \mathrm{GHz}$, most of the radiation comes from the arcsecond-to-mas components also visible in VLBI maps, with only secular changes in flux. Between 10-100 GHz the spectrum becomes dominated by radiation from the inhomogeneous relativistic jet (also called the core jet, the mas jet, or just the core), which appears to have long-term stability (e.g., Valtaoja et al. 1988, Lainela 1993). The flux variations on timescales from a few weeks to a few years are caused by evolving shocks in this relativistic flow. In most VLBI maps the jet and the newest shock appear as a single point source, the VLBI "core".

Is there room for other, as yet undiscovered, major components? The answer seems to be no. The quiescent high-frequency spectra above the turnover associated with the jet are steep and smooth (Valtaoja et al. 1988, Brown et al. 1989; Tornikoski et al. 1993; Gear et al. 1993), indicating that practically all the high frequency flux comes from the optically thin synchrotron jet.

\subsection{DATA VS. SHOCK MODELS}

The two main models for radio spectra and variations are the numerical code by Hughes et al. (1989), developed to explain the cm-wavelength flux and polarization variations observed in the Michigan monitoring, and the analytical model by Marscher and Gear (1985), which had its impetus in the observed high frequency behavior of $3 \mathrm{C} 273$. While both models assume that the cause of flux variations is the growth and decay of shocks in the jet, the Hughes et al. model is mainly applicable to the $\mathrm{cm}$-wavelength variations, and the MG model for shorter wavelengths.

The simple shock models predict three things. Since the flux variations are assumed to come from a new, and in the first approximation homogeneous, component, the variable component (i.e., the shock) should have a simple, homogeneous synchrotron spectrum with $\alpha_{\text {thin }}=2.5$. The time evolution of the spectral turnover $\nu_{\mathrm{m}}(t), S_{\mathrm{m}}(t)$ is model-dependent, but should show growth and decay stages. Finally, the observed maximum amplitude of the outburst, $\Delta S$ and the time delay $\Delta t$ should both have a definite (although model-dependent) dependence on $\nu$.

\subsection{The Shape and eVolution of The SHOCK SPECTRUM}

The first prediction has been tested by Valtaoja et al. (1988) and Lainela (1993), who used Metsähovi, Michigan and other data to construct quiescent and outburst epoch cm-to-mm spectra for a sample of sources. Subtracting the quiescent (i.e., the jet) spectra from the outburst spectra they found that within observational accuracy the shock does, indeed, have $\alpha_{\text {thick }} \approx 2.5$ and $\alpha_{\text {thin }} \approx-0.2$, indicating that the electron energy index $\gamma \approx 1.4$. 


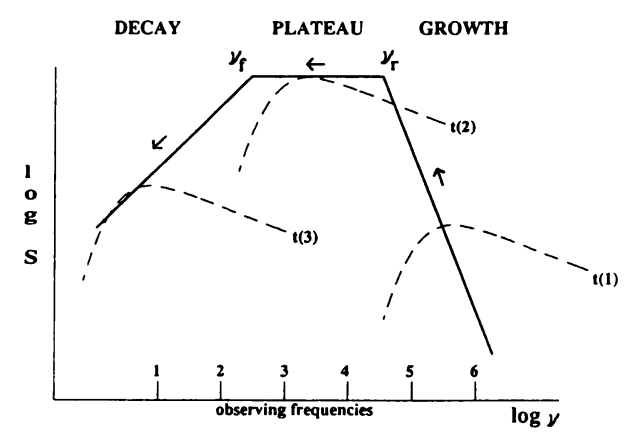

Fig. 1. Evolution of the shock spectrum in the generalized shock model. (Valtaoja et al. 1992a).

It is difficult to follow the time evolution of the turnover. Valtaoja et al. (1988) and Lainela (1993) combined essentially all available radio data in trying to follow the spectral evolution in a number of outbursts. The somewhat disappointing conclusion, also reached by Robson et al. (1993) in the case of the exceptionally well-monitored $3 \mathrm{C} 273$, was that the data agreed with shock models, but were not sufficient to validate them. On the other hand, no clear counterexamples, such as lower frequency variations preceding higher ones, were found.

\subsection{Flare amplitudes and time delays}

The maximum amplitudes of the outbursts and the delays between the times of maximum at different frequencies are much easier to observe, since they do not require simultaneous multifrequency data.

We have constructed a simple 'generalized' shock model for comparing the shock framework with observations (Valtaoja et al. 1992a). In this model, depicted in Figure 1, we have a simple homogeneous spectrum evolving in time through parametrized growth, plateau and decay stages. The evolutionary track $\left(S_{m}, \nu_{m}, t\right)$ must of course be obtained from specific models, but even without such models the predicted main features are readily seen. If the observing frequency is lower than the frequency of the plateau, one observes the adiabatic decay stages of the shock, with both $\Delta S$ and $\Delta t$ strongly dependent on $\nu$. If the observing frequency is 'high', the fluxcurves track closely each other, $\Delta t \approx 0$, and $\Delta S$ depends only weakly (as $\nu^{-\alpha}$ ) on frequency. Thus, for each outburst there is a critical observing frequency, below and above which two very different types of dependence are seen (Figure 2a).

Figure $2 \mathrm{~b}$ shows the results from the large Metsähovi and Michigan data sets. The $\Delta S(\nu)$ - and $\Delta t(\nu)$-dependences are in accordance with the shock framework. In particular, from the observed high frequency $\Delta S(\nu)$-dependence we recover the value $\alpha_{\text {thin }} \approx-0.2$, independently derived from the shape of the shock spectrum. 

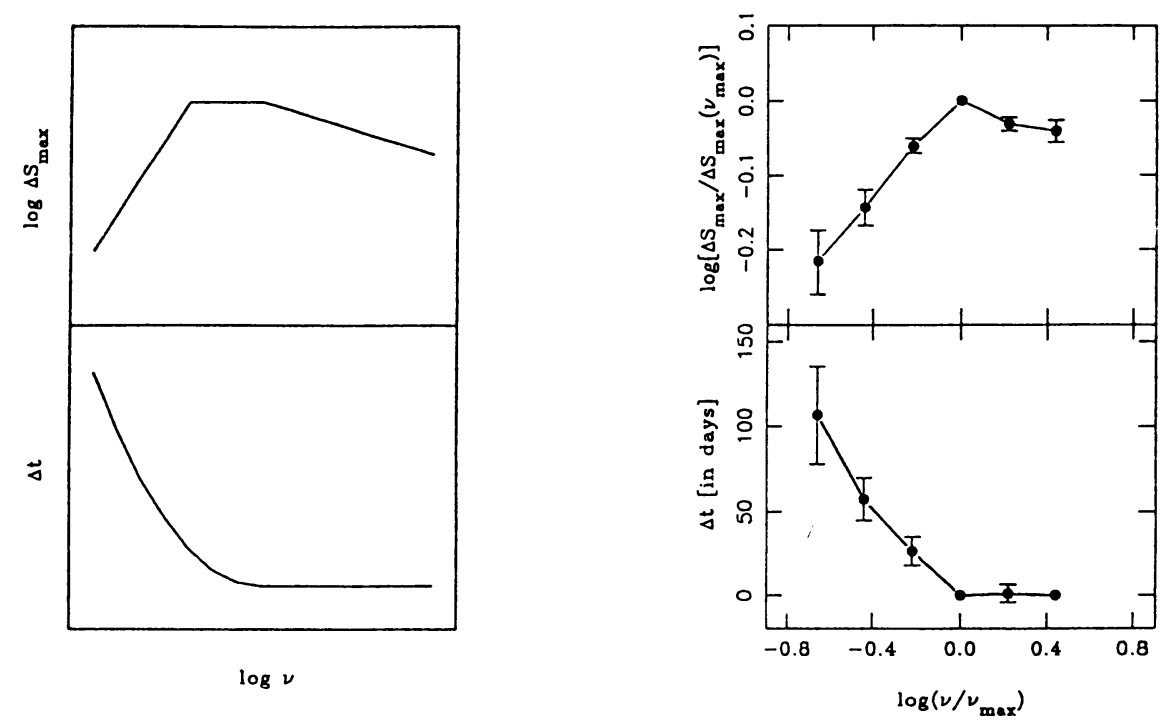

Fig. 2. The predicted and the observed (in a sample of $\mathbf{4 2}$ sources) dependence of burst amplitude and time delay on frequency (Lainela 1993).

\subsection{CONCLUSIONS FROM THE SPECTRAL AND VARIABILITY ANALYSIS}

The shocked jet scenario appears to be capable of explaining all the major radio variations in radio-loud AGN, whether they are radio galaxies, quasars, or BL Lac objects. In our sample of over 50 brightest sources we have found no clear evidence for other mechanisms, such as gravitational microlensing. Alternative mechanisms should stand out either in the detailed behavior of a single source, or in the statistical analysis of samples of sources; we have found neither.

The bad news is that the data are not sufficient to constrain shock models very strongly. It is perhaps not unfair to say that the observations are all compatible with the shocked jet scenario, but it would be difficult to single out any observational features which prove it to be valid. What is needed, among other things, are fully sampled mm-to-submm multifrequency fluxcurves, mm-polarization data, and improved theoretical models especially for the crucial growth stages of shocks.

\section{Variability, spectra and classification}

We can attach three separate sets of data to each radio-loud AGN: its classification (or rather, an overabundance of various possible classifications), its spectral characteristics (various spectral indices, turnover frequencies etc.), and its variability characteristics (maximum amplitude of variations, $\chi^{2}$-related parameters, timescales etc.). Using as complete and large data sets as possible, we may study 
the relations between these data sets.

An increasing number of such analyses have been published during the last few years (e.g., Valtaoja et al. 1988, Eckart et al. 1989, Ghosh and Gopal-Krishna 1990, Wiren et al 1992, Hughes et al. 1992, Aller et al. 1992, Valtaoja et al 1992b, Lainela and Valtaoja, 1993, Lainela 1993, Gear et al 1993, Teräsranta and Valtaoja 1993). By and large, the conclusions of these studies agree with each other, and conflicting results probably stem from the use of noncomplete samples. I will try to give a short summary of these results before considering their interpretation.

\subsection{The proper Classification of radio-loud AGN}

An impressive, if not outright depressive, number of AGN classifications have been proposed: steep/flat spectrum, VLBI compactness, core dominance, blazar/nonblazar, super/subluminal, OVV/ordinary, ad infinitum. The first task is to find out which divisions are reasonable, in the sense that they are based on intrinsic, persistent properties of the sources, and which divisions are fundamental, in the sense that they do not obviously derive from some other source properties. Much unnecessary confusion has been caused by neglecting to consider these things.

For example, it is common to divide radio-loud AGN into steep- and flatspectrum sources, usually using decade(s)-old catalogue data of spectral indices around a few $\mathrm{GHz}$ and an arbitrary dividing line $\alpha=-0.5$. It would be rather surprising if such a division would properly reflect some fundamental property of the sources, and indeed it does not, despite its widespread and continuous use.

It is not difficult to see why spectral steepness is not a fundamental division. Whatever the frequency range, the spectral index is obviously variable, and a 'steep-spectrum' source may change into a 'flat-spectrum' one, or vice versa, in a few years (e.g., Zhang et al. 1993). The low radio frequency radiation comes to a large extent from old, extended components with at best tenuous connections to the actual core regions. It is true that flat-spectrum sources are more variable than steep-spectrum ones (to take just one example), but this is simply due to the well-known fact that radio galaxies have on the average steeper spectra than quasars, and a flat-spectrum sample thus includes a larger fraction of quasars than a steep-spectrum one. Thus, the proper conclusion is that quasars are different (more variable, flatter spectra) from radio galaxies. The division into flat- and steep-spectrum sources does not add anything except confusion; one might as well mix apples and oranges in a basket, and then try to find out their average taste.

Similar criticism applies to most other groupings; I will mention only one more. If, as several lines of evidence indicate, the intrinsic Lorentz factors the flows in AGN are of the order 5-10, a simple calculation shows that even sources close to the plane of sky are superluminal. However, in such sources the shocks are Doppler deboosted, and probably below the detection threshold of VLBI. Thus, 'superluminal' is not a fundamental property of a source, but rather an observational indication that the shocks are bright enough to be detectable.

What then are the relevant classifications? In analyzing our data, we have tried 
all the above-mentioned (and quite a number of other) classifications. The conclusion, from several independent, large data sets, is the following:

All the statistically significant differences and correlations between the radio properties of $A G N$ are best explained by grouping the sources into radio galaxies, 'ordinary' quasars with low optical polarization ( $L P Q, p<3 \%$ ), and 'blazar-type' quasars with high optical polarization (HPQ, $p>3 \%$ ). BL Lacs form at best a marginally identifiable class of objects.

One might object that optical polarization is not any more observationally robust than the other indicators I have lambasted. While there is some justification in this criticism (e.g., the optical polarization often is highly variable, and some sources have high polarization produced by scattering), there is the well-known and persistent dichotomy between LPQs and HPQs, and sources once caught exhibiting $p>3 \%$ remain in the HPQ category forever (and usually behave like it).

\subsection{INFLUENCE OF PROPER CLASSIFICATION}

In order to convince the unrepentant, I give two random examples of how the choice of classification can crucially affect the conclusions. Aller et al. (1992) have studied the spectral properties of the Pearson-Readhead sample of radio galaxies, QSOs and BL Lacs. Using this division, they concluded that BL Lacs are different from quasars. However, by dividing the QSOs into LPQs and HPQs one finds that BL Lacs are different from ordinary quasars but similar to HPQs, leaving open the question whether they form a separate class of objects.

Gear et al. 1993) compare the mm-spectra of BL Lacs and flat-spectrum quasars, finding that they form two different classes. Again, using the LPQ/HPQ division and repeating their analysis, one finds the proper conclusion to be that BL Lacs are different from ordinary quasars, with the question of association between BL Lacs and HPQs left open.

\subsection{Properties of radio galaxies, LPQs, HPQs and BL Lacs}

Taking a grand average of the published analyses, the radio properties of these classes I have claimed to be fundamental can be shortly summarized as follows. For details, the reader is referred to the original papers listed in the beginning of this section; some examples are shown in Figures 3 and 4.

Highly polarized quasars have flatter spectra, are more variable, have shorter characteristic timescales and higher brightness temperatures than low polarization quasars (ordinary quasars). For all quasars, the spectral flatness and the amount of variability are positively correlated. Radio galaxies have the steepest spectra, are the least variable, have the longest characteristic timescales and the lowest brightness temperatures associated with outbursts.

$B L$ Lacs possibly have flatter spectra than quasars, but as a class they are not, repeat not, more variable than quasars, nor do they have shorter timescales or higher brightness temperatures than quasars. Many BL Lacs are among the least active and variable of all bright compact radio sources. In general, BL Lacs do not 


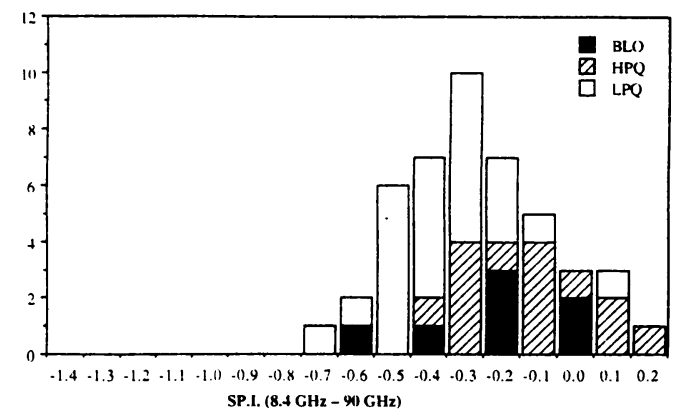

Fig. 3. Cm-to-mm spectral index in a complete sample of AGN (Tornikoski et al. 1993).

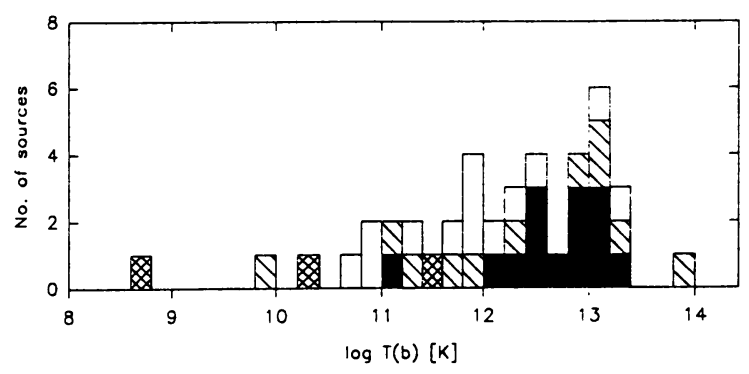

Fig. 4. The highest observed brightness temperature in a sample of HPQs (black), LPQs (white), BL Lacs (hatched) and radio galaxies (crossharched) (Teräsranta and Valtaoja 1993).

form a class clearly identifiable by their radio properties.

\section{Unified models?}

Is there a simple way to explain these results? Since one does know that the AGN will have different orientations relative to the line of sight, a natural way to proceed is to consider whether at least a part of the observations can be explained if orientation is assumed to be the decisive, fundamental underlying property. Of course, such an approach is highly simplistic - we do also know that sources have different intrinsic luminosities, different ages, different environments, etc. - but one may hope that in large enough samples these other factors will average out.

\subsection{SPECtra, VARIABILITy, AND ORIENTATION}

From the basic premise of radiation coming from a directed relativistic outflow and shocks immediately follow a number of testable predictions. As the viewing angle decreases, the Doppler boosting increases, the apparent luminosity increases, and the timescales are compressed, with the net result that the amplitude and the 
rapidity of variations increases.

With simple qualitative models (Eckart et al. 1989; Valtaoja et al. 1992a) the unified model predictions can be made more explicit. If the low-frequency radiation comes mainly from the unboosted extended component(s) and the high-frequency radiation from the boosted jet and shocks, the observed source parameters will depend on the boosting factor $D$ (i.e., the viewing angle) roughly as follows: the last turnover (=jet turnover) as $D$, the timescales as $D^{-1}$, the brightness temperatures as $D^{3}$ and so on. The low-to-high radio frequency spectral index and the different variability indices will also depend on $D$ in a more model-specific manner.

If we now, following Barthel (1989), identify the sources with largest viewing angles (least boosted) with radio galaxies, the intermediate sources as ordinary low polarization quasars, and the sources with smallest viewing angles as 'blazar'-type HPQs, both the differences between these classes and the correlations between, e.g., spectral flatness and variability, are explained very satisfactorily.

The fact that optical polarization is a good tracer of orientation is also easy to understand if we assume that a quasar core is surrounded by a dust torus (analogous to Seyferts), which hides the highly polarized optical core from view if the viewing angle is larger than the critical angle separating HPQs from LPQs. Although the optical polarization of a HPQ may occasionally drop to low values (when the core flux is small), the geometry of the source does not change and the classification based on polarization is robust.

We may even use the observed differences between LPQs and HPQs to estimate the relative amounts of Doppler boosting in these two classes of sources. Tornikoski et al. (1993) found that the last spectral turnover for LPQs occurs around $20 \mathrm{GHz}$ and for HPQs around 40-90 GHz. This then gives $D_{\mathrm{H}} / D_{\mathrm{L}} \approx 2-4.5$. Lainela (1993) used structure function analysis to derive characteristic timescales of 3.8-4 years for LPQs and 1.3-1.7 years for HPQs, giving $D_{\mathrm{H}} / D_{\mathrm{L}} \approx 2-3$; a reanalysis of the Michigan cm-data (Hughes et al. 1992) gives a value of $\approx 4$. Finally, Teräsranta and Valtaoja (1993) determined the brightness temperatures of outbursts (Figure 4), finding median values of $5.410^{12} \mathrm{~K}$ for HPQs and $8.110^{11} \mathrm{~K}$ for LPQs, consistent with $D_{\mathrm{H}} / D_{\mathrm{L}} \approx 2$. Thus, four idependent estimates indicate that HPQs have, on the average, 2-4 times more Doppler boosting than LPQs.

\subsection{VIEWING ANGLES OF RADIO SOURCES}

Different amounts of Doppler boosting can result either from different viewing angles or different intrinsic Lorentz factors; the correct alternative cannot be deduced from continuum data. But by combining continuum with VLBI we can do much better, and actually derive the viewing angles and Lorentz factors of individual sources from observed $(v / c)$ and $D \propto T_{b}{ }^{1 / 3}$ (Teräsranta and Valtaoja 1993).

If we make a plot of $(v / c)$ vs. $\log T$ (Figure 5a), unification schemes predict that the parent population will be found towards the lower left corner and the daughter population towards the upper right corner, with a similar range of $\Gamma$ but smaller $\theta$. In Barthel's unification scheme, the progression should be radio galaxies 

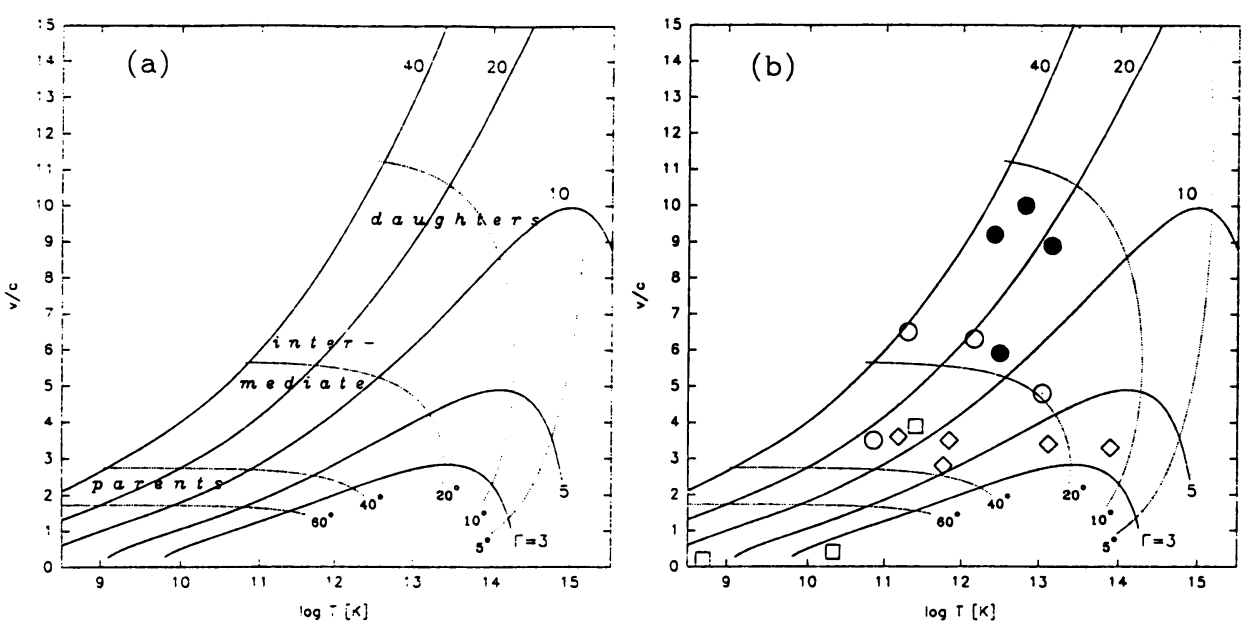

Fig. 5. (a) The relationship between the observable quantities $\left(v / c, T_{b}\right)$ and the intrinsic source parameters $(\Gamma, \theta)$. (b) The observed distribution (Teräsranta and Valtaoja 1993). Filled circles, HPQs; open circles, LPQs; squares, radio galaxies; diamonds, BL Lacs.

- LPQs - HPQs. If the BL Lacs come from a different parent population, they should occupy a different $(\Gamma, \theta)$-range.

In figure $5 \mathrm{~b}$ are plotted all the sources with sufficient VLBI and continuum data from Teräsranta and Valtaoja (1993). The statistics are small, but the distribution is as predicted. The estimated values are in good accordance with those derived from number counts, luminosity functions or other independent means. We find that a source appears as a HPQ if the viewing angle $\theta \leq 18^{\circ}$, as a LPQ if $18^{\circ} \leq$ $\theta \leq 35^{\circ}$, and as a radio galaxy if $\theta \geq 35^{\circ}$; for all, the Lorentz factors range between 10 and 35 (assuming $H_{o}=100 \mathrm{~km} / \mathrm{s} / \mathrm{Mpc}, q_{o}=1 / 2$ )

BL Lacs clearly occupy a different regime; they have $\theta \leq 40^{\circ}$ and $4 \leq \Gamma \leq 12$. The large viewing angles we derive for several BL Lacs are in accordance with the lack of rapid variability in most BL Lacs, and also with values derived from detailed model fittings. However, they do not agree with the small average viewing angles derived from luminosity function fits, where the assumption is that BL Lacs are beamed FR I radio galaxies.

\section{Conclusions}

The main conclusions derived from the analysis of high radio frequency spectra and variations are the following.

1) The framework of evolving shocks in a relativistic synchrotron jet is sufficient 
to explain the observations, without the need to invoke alternative explanations for either the spectral shape or the variations (with the possible exception of intraday variability, not discussed here). However, at present the observations provide only weak constraints for the shock models.

2) The unification of radio galaxies (FR II), ordinary low polarization quasars and higly polarized quasars is in accordance with, and almost certainly required by the data. In particular, the observed brightness temperature distributions of radio outbursts are very hard to explain without invoking orientation as the decisive factor differentiating radio galaxies, LPQs and HPQs.

3) The unification of radio galaxies (FR I) and BL Lacs does not find much support in the radio data. Several lines of evidence indicate that BL Lacs are not especially closely beamed. We favor the explanation that the 'complete samples' of BL Lacs used in various analyses are mixtures of two different populations, the nearby 'true' BL Lacs (with FR I parents) and the distant 'BL Lacs', which really are the extreme end of the HPQ population (FR II parents).

\section{References}

Aller, H.D., et al. 1985, ApJS 59, 513

Aller, M.F., et al. 1992, $A p J 399,16$

Barthel, P.D. 1989, ApJ 336, 606

Brown, L.M.J., et al. 1989, ApJ 340, 129

Eckart, A., et al. 1989, MNRAS 239, 381

Gear, W.K., et al. 1993, it MNRAS, in press

Ghosh, T., Gopal-Krishna 1990, A\&A 230, 297

Hughes, P.A., et al. 1989, ApJ 341, 54

Hughes, P.A., et al. 1992, $A p J$ 396, 469

Lainela, M. 1993, $A \& A$, in press

Lainela, M., Valtaoja, E. 1993, $A p J$, in press

Marscher, A., Gear, W.K. 1985, ApJ 298, 114

Robson, E.I., et al. 1993, it MNRAS 262, 249

Steppe, H., et al. $1992 A \& A S$ 96, 441

Teräsranta, H., Valtaoja, E. 1993, $A \& A$, in press

Teräsranta, H., et al. 1992, A\&AS 94, 121

Tornikoski, M., et al. 1993, AJ 105, 1680

Valtaoja, E., et al. 1988, $A \cup A$ 203, 1

Valtaoja, E., et al. 1992a, $A \& A$ 254, 71

Valtaoja, E., et al. 1992b, $A \in A$ 254, 80

Wiren, S., et al. 1992, $A J$ 104, 1009

Zhang, Y.F., et al., 1993, $A p J$, in press 